\title{
Tsafon
}

Revue d'études juives du Nord

$72 \mid 2016$

Juifs, Israéliens, dans la littérature française et israélienne

\section{Les cinq cents ans du Ghetto de Venise}

\section{Claude Cazalé Bérard}

\section{(2) OpenEdition \\ Journals}

Édition électronique

URL : https://journals.openedition.org/tsafon/369

DOI : $10.4000 /$ tsafon.369

ISSN : 2609-6420

Éditeur

Association Jean-Marie Delmaire

Édition imprimée

Date de publication : 1 décembre 2016

Pagination : 115-124

ISSN : 1149-6630

\section{Référence électronique}

Claude Cazalé Bérard, «Les cinq cents ans du Ghetto de Venise », Tsafon [En ligne], 72 | 2016, mis en ligne le 31 mai 2018, consulté le 26 juin 2021. URL : http://journals.openedition.org/tsafon/369 ; DOI : https://doi.org/10.4000/tsafon.369

Tsafon. Revues d'études juives du Nord 


\title{
Varia : Histoire
}

\section{Les cinq cents ans du Ghetto de Venise}

\author{
Claude Cazalé Bérard*
}

L'instauration du Ghetto de Venise par la Sérénissime République de Venise remonte très exactement au 29 mars 1516 : cinq cents ans plus tard il ne s'agissait certes pas de "célébrer » la naissance d'un système d'exclusion qui allait s'étendre à toute l'Europe, en donnant son nom à tout lieu de discrimination et de ségrégation, avec toutes les variantes inventées par l'imagination perverse de pouvoirs antisémites, jusqu'aux pogroms et à l'extermination conduisant à la «solution finale » programmée au $\mathrm{XX}^{\mathrm{e}}$ siècle par le nazisme. De fait d'autres formes de séparation existaient déjà (au Maroc, en Tunisie, en Algérie, en Égypte, en Espagne, Juderias, dans les pays germaniques, Judengasse) mais avec le Ghetto de Venise on assiste pour ainsi dire à une institutionnalisation formelle de ce type de ségrégation.

Les manifestations aux larges résonances internationales, qui sont appelées à se succéder de mars à décembre 2016 (colloques et séminaires scientifiques, expositions photographiques, représentations théâtrales, concerts...), offrent plutôt l'opportunité de faire le point sur la vie actuelle de ce quartier de Venise (hors des circuits touristiques les plus fréquentés), sur l'histoire séculaire d'un rayonnement intellectuel, artistique, spirituel qui s'est exprimé en dépit des conditions de sujétion, et sur les acquis les plus récents de la recherche concernant les phénomènes d'exclusion, d'enfermement, de marginalisation, dans une perspective européenne et plus largement globale (ce que signale le Séminaire international organisé par l'Université de Princeton: «Le

\footnotetext{
* Université Paris Ouest Nanterre La Défense.
} 
Ghetto comme métaphore globale »). D'où également de nouvelles publications sur le sujet (voir la bibliographie en fin d'article).

La particularité du Ghetto de Venise et de sa communauté ne tient pas seulement à son antériorité mais à son caractère à la fois d'insularité et d'extraordinaire ouverture sur le monde. Ce furent tout d'abord des Juifs allemands et italiens qui s'installèrent à Venise, suivis par des levantins (Juifs sépharades provenant d'Istanbul, de Salonique et d'autres ports méditerranéens). Il faut attendre la fin de la guerre de Chioggia (1381) pour qu'une première condotta (contrat) les autorise à résider officiellement à Venise (mais sans accès à la propriété) : ce contrat renouvelé d'abord tous les cinq ans, puis tous les dix ans, définissait leur statut juridique, leurs droits et les impôts à payer à la République. Cette population s'adonnait initialement aux métiers liés aux prêts d'argent, au commerce d'objets et vêtements usés (strazziaria), tout en maintenant des liens étroits avec les communautés d'origine, d'Orient et d'Occident. Ainsi, au $\mathrm{XVI}^{\mathrm{e}}$ siècle, affluent à Venise des Juifs fuyant l'Espagne et le Portugal, ainsi que ceux habitant la terre ferme chassés par les guerres menées contre Venise par la Ligue de Cambrai : généralement riches et d'un niveau social élevé, ils apportaient des capitaux dont la République avait le plus grand besoin. À son plus fort niveau d'expansion, la communauté juive atteignit jusqu'à cinq mille personnes. De façon tout à fait pragmatique la République fit en sorte de fixer cette population qui lui permettait de s'enrichir, tout en la tenant sous un étroit contrôle : un décret du Sénat vénitien (29 mars 1516) stipula que les Juifs devaient se regrouper dans une seule zone de la ville, le quartier de San Girolamo, au nord de Venise, où se trouvaient les anciennes fonderies (gettare signifiait «fondre », d'où geto vecchio, l'ancienne fonderie, geto nuovo, la nouvelle, gheto avec la prononciation à l'allemande de ses habitants) : le quartier assigné n'aurait que deux points d'accès au reste de la ville, dont les portes se refermeraient la nuit et dont la surveillance devrait être assurée par quatre gardiens payés par les Juifs eux-mêmes; deux embarcations du Conseil des Dix devraient en outre circuler toute la nuit sur les canaux entourant le quartier. Les Juifs devaient porter un signe distinctif : un rond jaune, puis un turban ou un chapeau d'abord jaune puis rouge. Moyennant quoi les Juifs pouvaient pratiquer le prêt à usure et le métier de fripier.

La création du Ghetto Nuovo (auquel s'ajoutèrent en 1541 le Ghetto Vecchio et en 1633 le Ghetto Nuovissimo) marquait cependant un tournant significatif dans 1'histoire des communautés juives : à compter 
de cette date, après tant d'expulsions, de conversions forcées, de discriminations de toutes sortes, les « ghettos » allaient se multiplier dans toute l'Europe, même si leur création eut pour effet de stabiliser l'installation des familles juives, moins sujettes du coup à de subits refus de séjour. La condotta de 1589, reconnaissant la liberté religieuse, accorda aux Juifs de Venise des privilèges commerciaux (le négoce international où ils excellaient rapportait à la République) et davantage de liberté dans les activités (ateliers, associations de bienfaisance, théâtres...), alors que les professions publiques, les universités, la participation à la vie politique leur étaient niées (même si les médecins juifs fort recherchés pouvaient circuler librement); toutefois l'augmentation continuelle des loyers et des impôts n'en créa pas moins des conditions de très grande pauvreté.

D'autre part, si la République de Venise tenait fortement à son autonomie vis-à-vis de l'Église et à sa modération religieuse, l'Inquisition veillait. Et la vie quotidienne était tout de même marquée par des vexations ou des arrestations arbitraires : ainsi, un Mordechaï ben Menchem Baldosa fut assassiné sans jugement en 1672. Ce qui fait que l'on ne peut pas voir, en ces siècles où la Sérénissime donnait l'image de sa splendeur et de sa florissante civilisation, une période particulièrement heureuse de l'histoire de la présence juive en Italie. Dès que furent abattues les portes qui limitaient l'accès au quartier, en juillet 1797, le Ghetto qui était né comme un espace de ségrégation se transforma en un quartier où les Juifs aimaient librement s'installer et se réunir, même si la communauté ne comptait plus que 3.000 personnes. Ce n'est qu'avec l'Unité italienne que les Juifs de Venise obtinrent une égalité complète des droits.

Ainsi, contrairement à Rome où le ghetto imposa des conditions de pauvreté et de ralentissement dans le développement social et culturel, les mêmes contraintes à Venise provoquèrent le phénomène contraire, en encourageant la population à préserver son identité tout en assimilant les connaissances et les expériences du monde environnant extrêmement diversifié et changeant dans une ville aussi active économiquement et culturellement, qui était un carrefour entre Orient et Occident, entre les nations du pourtour de la Méditerranée et de l'Europe du Nord. Ce qui fait que Venise fut - en dépit des contraintes matérielles imposées - un des centres les plus brillants de la culture juive en Europe. Dans le Ghetto de Venise, on construisit de splendides synagogues (où étaient pratiquées des liturgies différentes d'origine ashkénaze, sépharade, italienne)- 
jusqu'à neuf synagogues, appelées schole, en 1719 (Schola Grande Tedesca, Schola Italiana, Schola Levantina, Schola Provenzale, Schola Spagnola o Ponentina...) - très discrètes à l'extérieur elles se distinguaient à peine des hautes habitations serrées les unes contre les autres en raison de l'espace restreint; on y ouvrit de célèbres écoles rabbiniques et on y imprima les premiers exemplaires du Talmud et de bien d'autres ouvrages en langue hébraïque. La communauté compta des personnalités marquantes : de savants rabbins comme Isaac Abravanel (1437-1508), philosophe et homme politique d'origine portugaise, Léon da Modena (1571-1648) maître en théologie et poète, musicien, auteur de théâtre, et Simon Luzzatto (1580-1663) enseignant et fameux polémiste, écrivain, libraire, commerçant ; la célèbre poétesse et intellectuelle Sara Copio Sullam (1589/90-1641) qui tint un salon littéraire fréquenté par l'aristocratie vénitienne. Au cours du $\mathrm{XIX}^{\mathrm{e}}$ siècle et au début du $\mathrm{XX}^{\mathrm{e}}$, le quartier, considéré comme pauvre et dégradé, se vida progressivement de ses habitants au profit d'autres quartiers de Venise. Ce n'est que vers les années soixante-dix du $\mathrm{XX}^{\mathrm{e}}$ siècle que le Ghetto trouva un nouvel essor grâce à une entreprise de sauvegarde des monuments anciens et à l'afflux des touristes (Musée, Bibliothèque, Archives) : ainsi un monument (Basreliefs de Blatas sur le Campo du Ghetto Nuovo) y a-t-il été installé en commémoration des 240 Juifs déportés pendant la Deuxième Guerre mondiale.

La communauté juive de Venise compte aujourd'hui un peu moins de 500 personnes, mais elle conserve ses traits d'homogénéité et de cohésion dus à sa grande stabilité et à sa capacité de résistance dont témoigne encore l'usage du judéo-vénitien: dialecte incompréhensible pour les non initiés, mais depuis toujours un moyen de reconnaissance et de protection. Même si le Ghetto de Venise s'est dépeuplé, si les boutiques sont moins nombreuses, des artisans et des artistes restent fidèles à la tradition et continuent à offrir leurs créations originales.

Pour finir, de lieu d'exclusion à lieu de rencontre, le Ghetto peut apparaître malgré tout comme le symbole de la survie d'une minorité, de la sauvegarde d'une identité tandis que continuait à se développer une tradition hébraïque qui sut s'enrichir au cours des siècles de l'apport de Juifs de toutes provenances contribuant au renouvellement constant des formes de vie, de pensée et de culture. Une culture basée sur l'écoute, le dialogue, la mémoire, la tolérance, le respect de la vie et de la nature, la confiance en l'homme, la liberté fondée sur la reconnaissance de l'autre, qui allait en retour influencer la culture italienne et européenne. 


\section{Bibliographie}

- Calimani Riccardo, Histoire du Ghetto de Venise, préface d'Élie Wiesel, Paris, Taillandier - Texto, 2008 (Stock, 1988).

- Calabi Donatella, Venezia e il Ghetto, Torino, Bollati Boringhieri, 2016.

- Fortis Umberto, L'attività letteraria nel ghetto (1550-1650), Livorno, Salomone Belforte Editori, 2015.

L'Histoire a consacré son numéro hors série, d'avril 2016, à «Venise, la cité monde », il renferme deux articles sur le Ghetto de Venise :

- Chauvard Jean-François, "Ghetto : histoire d'un quartier réservé », pp. 48-51.

- Calabi Donatella, « Les Juifs dans la ville », pp. 52-55.

Et le $\mathrm{n}^{\circ} 427$ de septembre 2016 publie un article dans lequel le Ghetto de Venise est largement présenté :

- Gasperoni Michaël, «1516, naissance des ghettos », pp. 13-21.



Passage d'entrée au Ghetto Nuovo (Gheto novo) (Photo Claude Cazalé Bérard) 


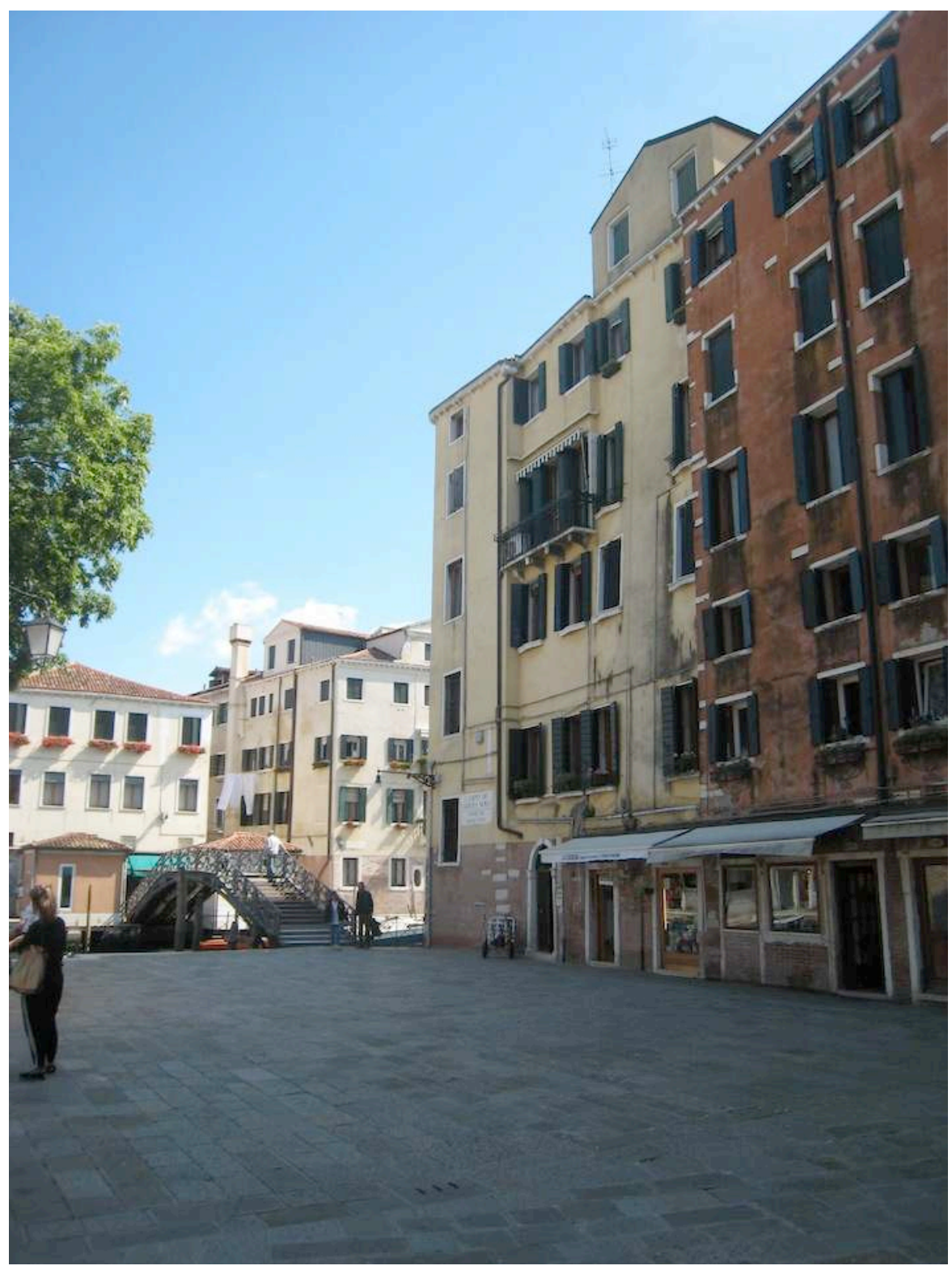

Le Campo

(Photo Claude Cazalé Bérard) 


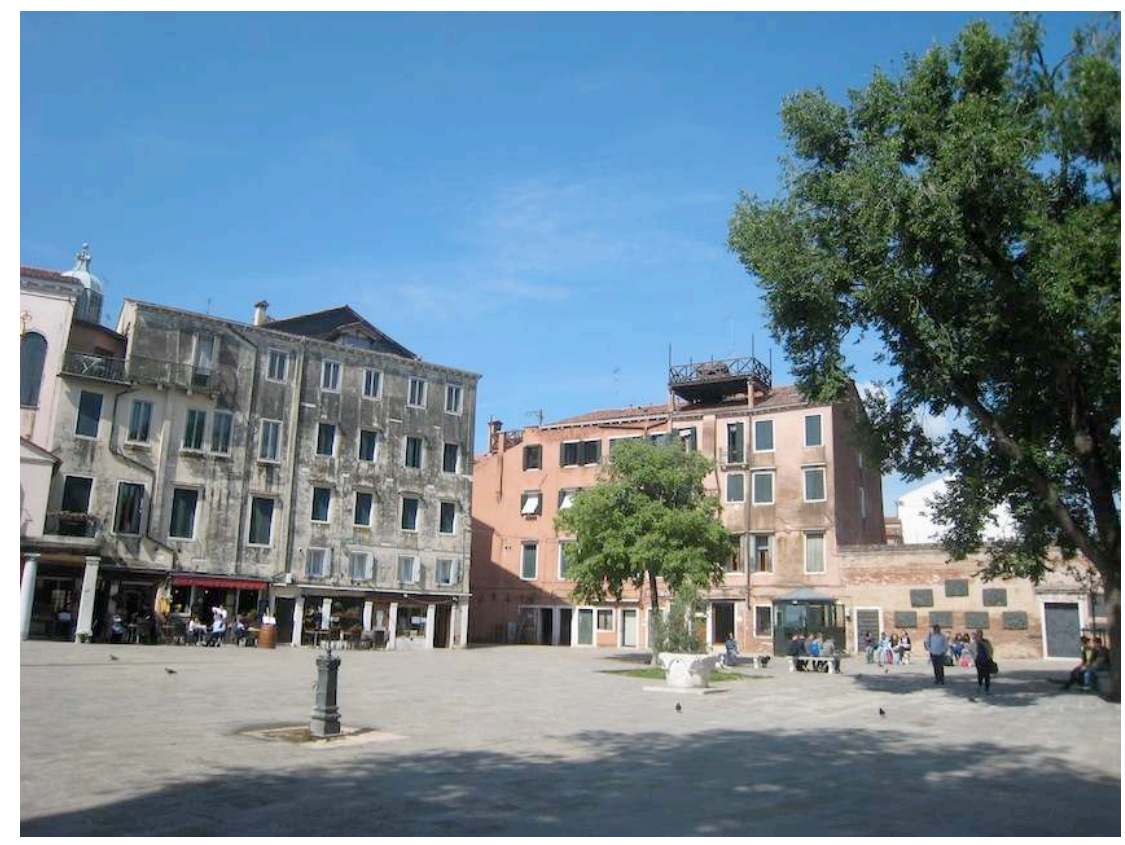

\section{Le Campo}

$\mathrm{Au}$ fond le monument de Blatas avec les plaques commémoratives (Photo Claude Cazalé Bérard)

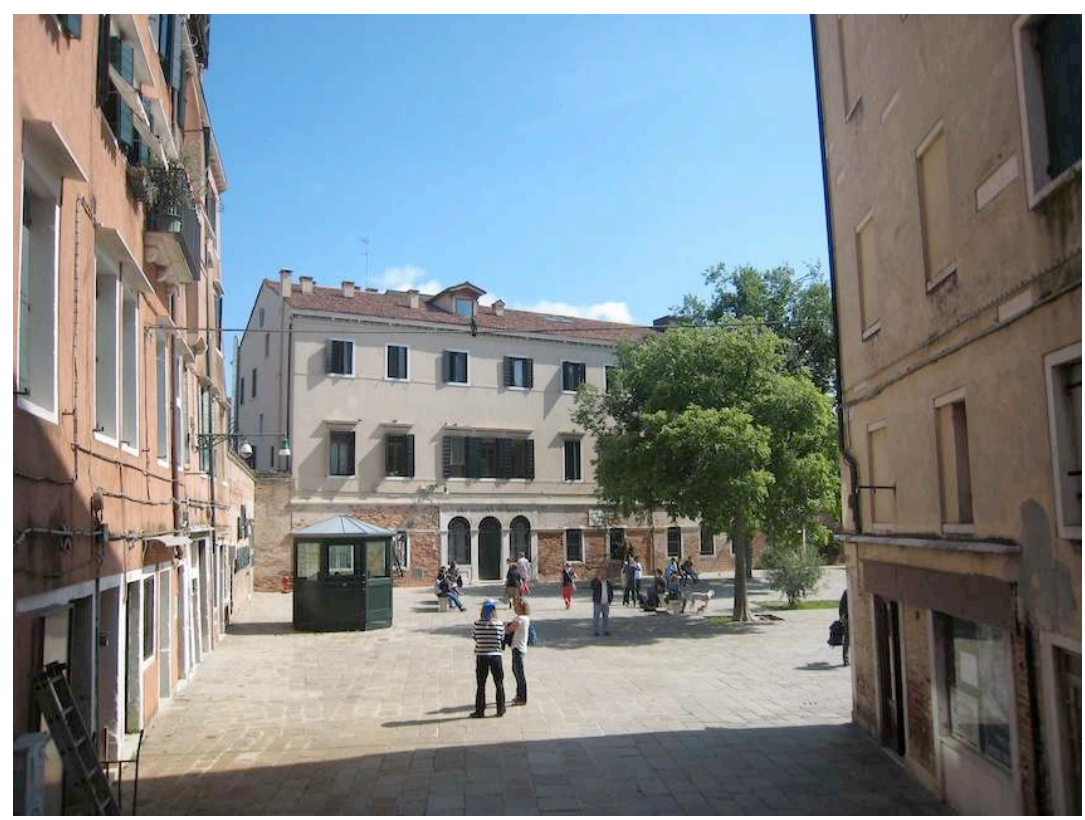

Campo du Ghetto Nuovo (place principale)

(Photo Claude Cazalé Bérard) 


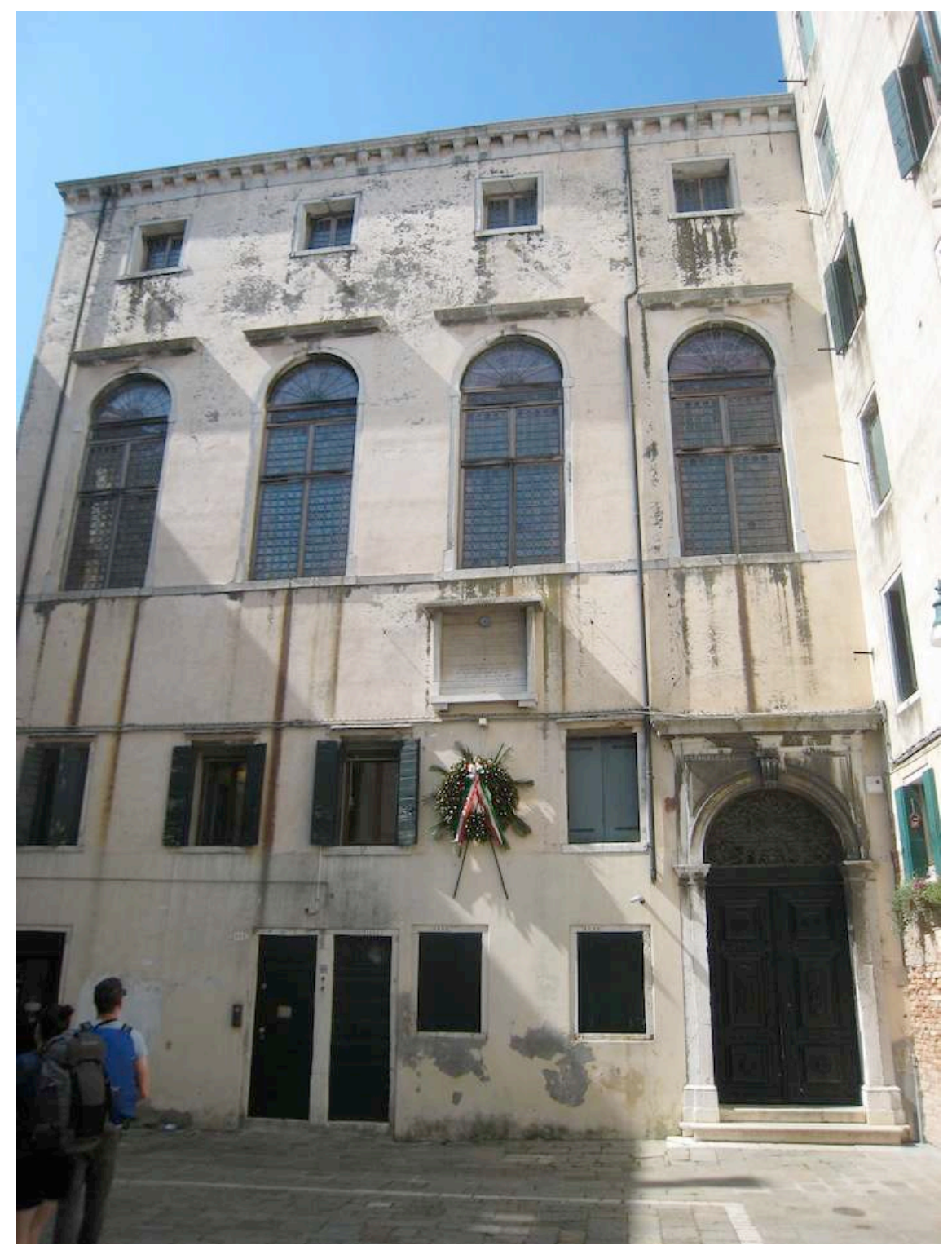

Façade de la synagogue espagnole du Ghetto Nuovo (Photo Claude Cazalé Bérard) 


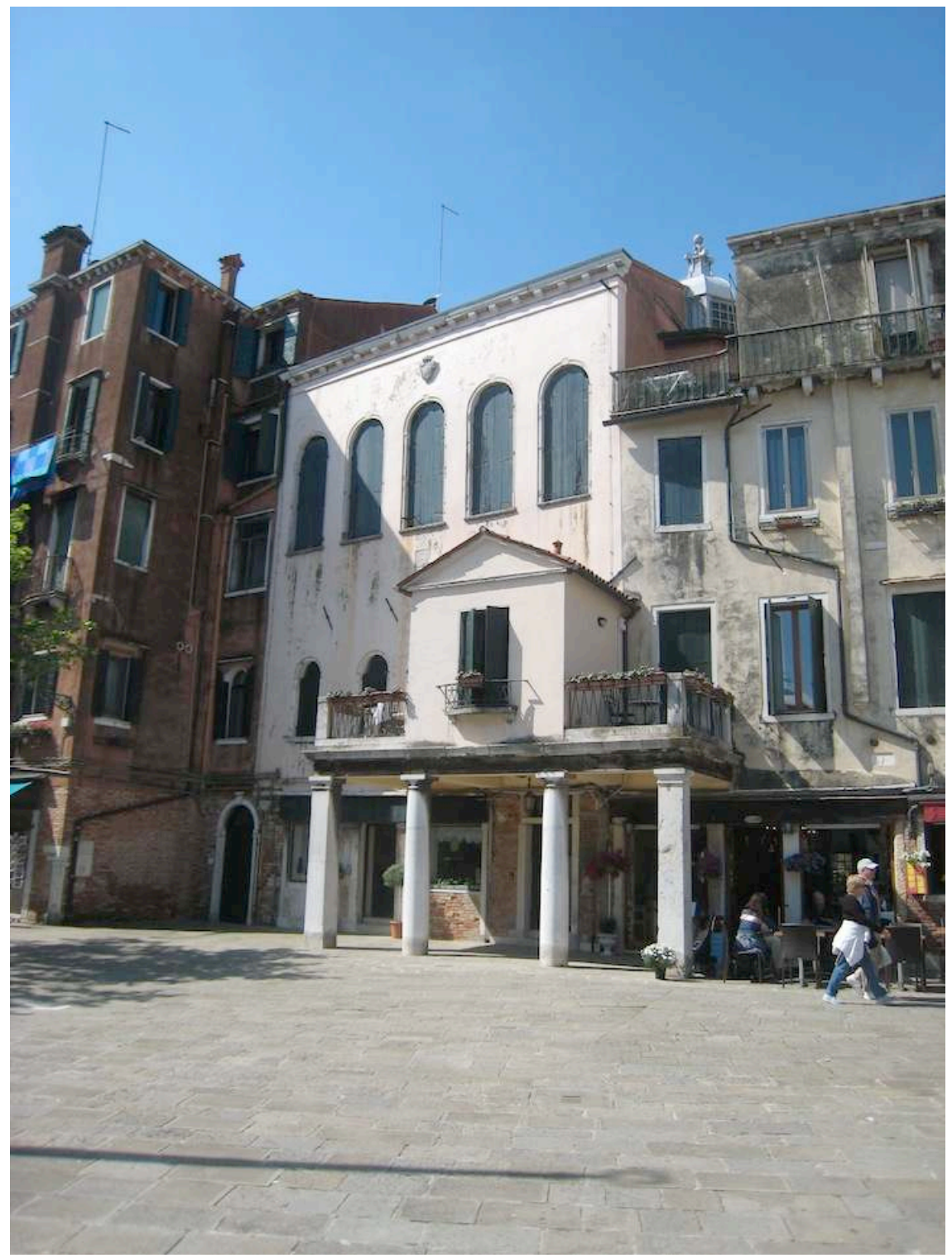

Schola italiana (façade synagogue italienne)

(Photo Claude Cazalé Bérard) 


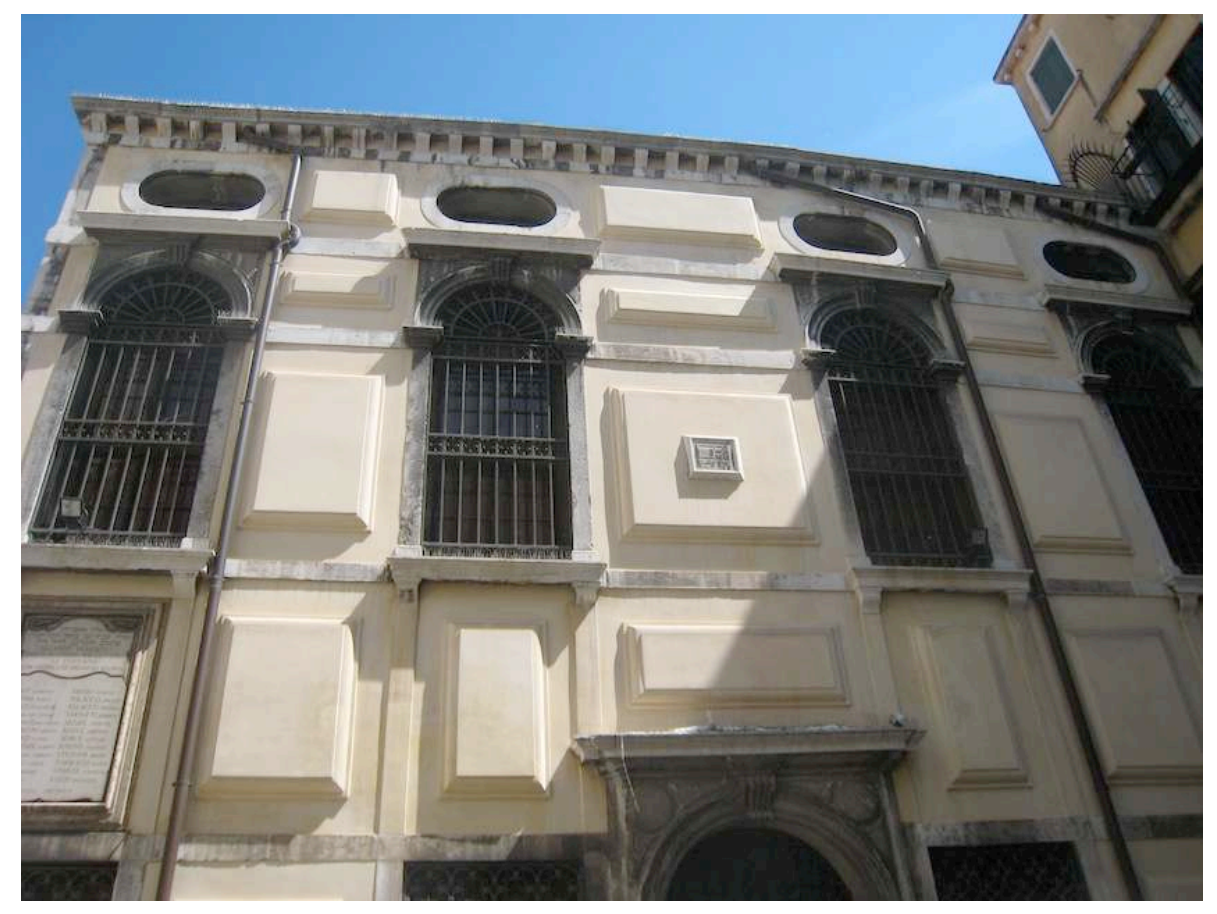

Façade d'une synagogue, Campiello delle schole (Photo Claude Cazalé Bérard) 